FUTURE PROSPECTS OF THE TPC IDEA. D. R. Nygren* (Lawrence Berkeley Laboratory, University of California, Berkeley, California 94720). 29.90. Other topics in high-energy and nuclear experimental method. and instrumentation

\title{
ABSTRACT
}

Future Prospects of the TPC Idea. D. R. Nygren (Lawrence Berkeley Laboratory, Untverstty of California, Berkeley, California 94720). Physıca Scripta (Sweden).

General aspects affecting TPC size, readout $p$ lane characteristics and operation in the LEP environment are presented, with the general conclusion that modest improvements relative to PEP-4 can be realized in several areas. The problem of positive ion reduction is discussed according to two qualitatively new operating modes, asynchronous and synchronous gating. Either gating mode appears to offer a means to eliminate almost completely the ion return flux. Some speculative ideas involving 3-component gas mixtures, 10w-mass components and parallel plane geometry are presented as future possibilities.

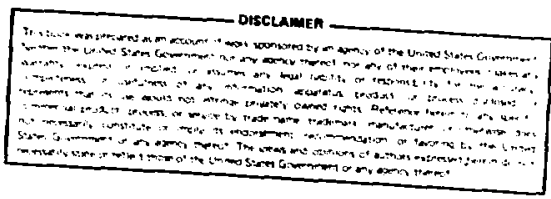

*This work has been supported by the High Energy Physics Division by the U. S. Departwent of Energy under contract No. W-7405-ENG-48. 
1. General Characteristics of a TPC Detector for LEP Energies

\subsection{Scaling from PEP-PETRA Energies to LEP}

The facility based on the TPC now under construction at PEP provides a useful frame of reference for beginning the discussion of future prospects (I). The relevant characteristics of this apparatus are given in Table 1 along with corresponding parameters for a hypothetical version scaled for LEP. The scaling requirements for momentum resolution are straightforward if the assumption is made that observed charged particle multiplicities will continue to rise $\simeq \sqrt{\mathrm{E}_{\mathrm{cm}}}$ (2). This implies that the mean momentum $\langle P\rangle$ of the charged secondaries should also rise as $\sqrt{E_{\mathrm{cm}}}$. Comparable mean momentum resolution is obtalned by scaling the radius of the active TPC volume by the ratio

$$
\frac{R_{\text {LEP }}}{R_{P E P}}=\left(\frac{E_{\text {LEP }}}{E_{P E P}}\right)^{\frac{3}{4}}=1.5
$$

for constant magnetic field.

The requirements for $d E / d x$ resolution needed for particle Identification do not exert as strong an influence on the choice of radius as do other factors. The rate of the relativistic rise and the onset of the transition to the Fermi plateau both vary logarithmically with gas density. Consequently, only rather modest gains are obtained even with substantial reductions in gas pressure. As tine product of the total sampling length $\times$ pressure should be held at approximately 10 meter atwospheres (for 90-10 Argon-methane) to maintain adequate resolution, the choice of radius is determined essentially by the rapid dependence of costs and momentum resolution. The operating pressure is then chosen to weet the criterion established for $\mathrm{dE} / \mathrm{dx}$ 
resolution. The choice in Table 1 for outer radius is taken to satisfy Eq. (1) and leads to a minimum pressure of 6.3 atmospheres. The value of pressure indicated in Table $1,7.6$ atm., was chosen to reach a $\mathrm{dE} / \mathrm{dx}$ resolution of $5.0 \% \mathrm{FWHM}$, using the calculations of Allison and Cobt (3). For these conditions the data of Walenta et al. Indicate a Fermi plateau of $1.35 \times \mathrm{dE} / \mathrm{d} \mathrm{x}_{\min }$ and an onset of the density effect at $50 \mathrm{GeV} / \mathrm{c}$ for kaons $(4)$.

With some degree of arbitrariness, the maximum drift length has been increased from $100 \mathrm{~cm}$ to $120 \mathrm{~cm}$ for improved solid angle and momentum resolution for tracks at angles less than $45^{\circ}$ from the beam direction. Indeed, a true optlmization might lead to smaller values since the high voltage requirements (and the attendant nonlinear aggravations) is linearly related to the maximum drift length. However, the reduced pressure more than offsets this increase, leading in fact to a smaller high voltage requirement for LEP than for PEP.

\subsection{Characteristics of the Readout Planes}

The number of sectors has been increased from 6 at each end to 8 , motivated primarily by the need to maintain electrostatic stability for the longest sense wire. This choice also reduces a contribution to the spatial resolution (caused by ionization fluctuations) which enters whenever the projection of the track Image in the readout plane is not perpendicular to the sense wire direction. Therefore an increase in wire spacing from 4 mim to $4.6 \mathrm{~mm}$ can be introduced without penalty in resolution. The increase to $4.6 \mathrm{~mm}$ and the reduction in operating pressure both act to improve further the margin of electrostatic stability, more than offsetting the increased length of the longest sense wire. These choices lead 
to 256 sense wires per sector.

Further improvement in spatial resolution can be achieved by reducing the dimension of the cathode pad along the sense wire directtion. The measured response of the pad geometry for PEP-4 is shown in Fig. 1, an approximately gaussian shape with width determined mainly by the distance between cathode and sense planes (5). The effects of electronic noise must be included, and the pad geometry for PEP-4 leads to a scalloped spatial resolution depending on position along the sense wire as shown in Fig. 2a. Reducing the pad dimension from $8 \mathrm{~mm}$ to $5 \mathrm{~mm}$ practically eliminates this effect, Improving both the spatial resolution and track pair resolution in this almension as shown in Fig. 2b. The pad signals for similar wire gain would be smaller, making it necessary to increase the wire gain by 25 to $50 \%$ relative to PEP-4. The economic impaci of this choice for pad dimension would be substantial, and a true optimization including cost may lead to a rather different configuration with perhaps fewer than 16 pad rows.

\subsection{Performance Characteristics}

The resolution in $x$, along the sense wire, is determined by contributions from several sources such as diffusion, ionization fluctuations, electronic noise, and various sorts of systematic errors, drifts, mechanical tolerances, and calibration nonlinearities. Data taken with $1.8 \mathrm{GeV} / \mathrm{C}$ protons traversing a TPC prototype yielded $100 \mu \mathrm{m}$ rms resolution (1imited by systematic calibration errors of the prototype electronics) in good accord with expectations (6). $150 \mu \mathrm{m}$ should be an achievable resolution for the LEP IPPC.

The track pair resolution in $x$ depends upon the criteria adopted for tolerable interference, and may depend upon factors such 
as track momentum and dip angle, need for unambiguous $d E / d x$ information, event category, etc. For track reconstruction it is perhaps sufficient to take advantage of the fact that quite good resolution can be obtained by taking the ratio of signals from two adjoining pads. As shown in Figure 3, two tracks separated by $15 \mathrm{~mm}$ in $x$ and arriving simultaneously with similar levels of ionization produce a bimadal pad signal distribution involving six or seven pads. The signals observed on the left and right outermost pad pairs would he essentially unmixed due to the very rapid falloff of the pad response. The value $15 \mathrm{~mm}$ taken as the boundary for track pair interference is conservative since fitting techniques should be successful even when mixing is significant.

In $z$, the drift direction, the resolution is again limited by diffusion, ionization fluctuations, etc. With the TPC prototype referred to above, data were also taken simultaneously with a 16channel prototype CCD readout system. The CCD system recorded the time structure of 16 wire signals. A simple three point algorithm reconstructing mean drift time to each wire gave a resolution of $150 \mathrm{Lm}$ rms (for drift lengths of a few $\mathrm{cm}$ ), and more sophisticated approaches using all available information should approach the diffusion limit. This resolution in $Z$ is considerably better than needed for momentum resolution, but is quite beneficial for the resolution in reconstructed invariant mass. 
The track pair resolution in $Z$ may be improved by using higher sampling rates, e.g., I4MHz $\rightarrow 25 \mathrm{MHz}$. The criterion adopted here for adequate separation in $Z$ is that at least 8 sampling periods should elapse between track signals. The extent of overlap is shown in Fig. 4, and corresponds to a 18 rN resolution in 2 for $L E P$ TPC. Therefore, a rough zone of track-pair interference can be defined as

$$
\Delta x \Delta z=15 \times 18 \mathrm{mM}^{2} .
$$

In Figure 5 the calculated momentum resolution is shown, using the parameters given in Table 1 for LEP TPC.

The total number of samples in drift time is 512 , given by the maximum drift time divided by the clock frequency. The CCD used in PEP-4 is the Fairchild 321A, a dual channel device with 455 cells per channel, a signal-to-noise rativ in excess of $55 \mathrm{db}$, and clocked at 13-15 MH 2. Much higher clow rates are possible, but the task of designing clock drivers for 16 channel boards becumes increasingly difficult as frequency increases. The total number of cells, 455, places an upper limit of $20 \mathrm{MHz}$ for this device in tr:s application in order to span the entire drift length (neglecting trigger formation time).

Recently, the prospect for inexpensive 6-bit $20 \mathrm{MHz}$ flash encoders has become more likely (7). Two such devices cascaded with different sensitivities should provide a good match to the signal processing requirements both for high resolution in the pedestal and small pulse height range and for large dynamic range. The use of flash encoders necessarily entails a considerable array of ultra high speed memory for each channel. At this time the choice between CCD or flash encoder techniques is not clearly established, as both technologies are evolving rapidly. 
The preceding discussion illustrates some modest performance improvements appropriate for the LEP environment. However, it may be important or even essential to implement a qualitatively different mode of operation in order to eliminate electrostatic distortion induced by space charge effects of positive ions drifting back from the readout planes.

\section{Gated Mode of Operation}

The large volume of the TPC drift region makes the TPC technique particularly vulnerable to ristortions of the electrostatic field due to collective space charge effects of positive ions migrating from $t i \ldots \ldots \ldots$ wires back to the central high voltage membrane. This sensitivity is exacerbated in the LEP TPC because of the larger dimensions. The amount of ionizing radiation trlerable without perceptitile diatortion is quite small, on the order of a few Gel/sec deposited within the artive volume. As background fluxes are notoriously difficult to predict or eliminate in practice, it is prudent to implement in advance sone scheme for eliminating or reducing substantially the return flux of positive ions. Two variants of a gated mode of operation appear to bo interesting possible solutions. Both take advantage of "electronjc shut tex" concepts developed many dccades ago in the field of gaseous electronics and recently incorporated into MPC detectors by Charpak and Sauli $\{8\}$. Figure 6 a shows a cross-section of the TFE readout plane with typical geometry. Dashed lines indicate the trajectories electrons follow toward the anocies. Because ion diffusion is small and becawse the aralanches do not spread arrund alle wire, positive ions tend to fallow the parent ejeciron trajectories back toward the drift region and tore than a third of the total pas through the grid. Figure $6 \mathrm{~b}$ shows the same geacetry hut with alternate polarity voltages imposed on 
the wires of the grid plane. In this electrostatic configuration, the shutter is closed, and nelther electrons nor positive lons can traverse the grid plane.

The generation of positive ions is an inevitable consequence of the proportional multiplication process at the sense plane anodes. The strategies to eliminate the subsequent space charge effects can involve a combination of 1) producing positive ions only when trigger signals indicate that a good event candidate has occurred; 2) preventing the return of positive lons to the drift region by capitalizing on the very large differences between electron and ion mobilities.

\subsection{Asynchronnus (reted Grid Mode}

This mode of operation maintains the grid in the closed stats until a prompt crigger dorision (most likely based on caiorimetry) indicates a gnod event ra.ıdidate. Then the grid must be "opened" as rapidly as possible since information is drifting onto the grid at several $\mathrm{cm} / \mathrm{usec}$. To open the grid rapidly and with sufficient pulst symetry that the grid pulsing does not drive the sensitive preamplifiers into deep saturation is a nontrivial problem.

After the electrons in the TPC drift region have been swept out ( $=20$ usec), the shutter can be closed easily before the ions produced in the avalanches at the sense wires arrive in the grid region. Dispersion in arrival time of the ions at the grid plane 1 s dominated by the variations in path length and $:$ field to the negatively charged grid element (see Fig. 6b), and typical migration times are $900 \mathrm{ssec}$. By arranging that the grid always stay closed whenever the ions have drifted into the grid vicinity, the positive ion return flux can be reduced by a factor of at least several hundred. The only 
and secondary positive ions that escape the grid during the 25 usec open period, having their origin in avalanches at the sense plane from earlier tracks which traversed the readout plane when it was closed. Whether or not any positive ions can travcrse the grid during a 20 usecond "exposure" depends on geometry as well as operating conditions and may turn out to be a very smal! fraction. It is therefore conceivable that in this mode of operation the fundamental limit to tolerable background will be the primary tonization left by the high energy particles in the drift region.

In the asynchronous mode of operation the generation of sufficiently clean triggers ( $<\mathrm{KHz}$ rate) in the smallest possible fraction of a usecond is certainly a formidable challenge, but the most difficult task is the development of a triggerable readout plane. The ideal transition of the grid from the closed to the open state would require both polarity offset voltages $\pm \Delta v$ to ramp within a few hundred nanoseconds in perfect symetry to zero with the absence of transient effects. Deviations from this ideal would necessarily induce signals on both pad and wire preamps, and could be of either polarity. As an illustration of the sensitivities involved, the PEP-4 TPC electronic circuitry has noise characteristics of a few tiundred electrons rms and preamplifier charge sensitivicies of $=10^{12} \mathrm{~V} /$ coulomb. The preamplifiers have a long time constant, 6 usec, and can absorb a few dozen tracks in a few microseconds before nonlinear response appears. Each track produces $=0.05$ picocoulombs at the wire, which gay be compared with $\$ 250$ picocoulombs that would be induced if ail grid wires were to receive a single polarity 100 volt pulse. Therefore, the required symetry of the grid pulsing in order to 
cancel out the induced charge to a level equivalent to one or a few tracks would be ap.roximately $2-5$ parts in $10^{4}$.

However, in the gated mode there is less motivation to operate with the lowest possible wire gain, since the positive ions are essentially eliminated. More wire gain would allow correspondingly less sensitive wire preamplifiers to be used, and this will reduce the impact of the grid signals proportionally. As the wire gain is increased the response to incldent electrons will eventually exhibit saturation, artificlally reducing the relativistic rise. The saturation effects are dependent upon the relative orientation of track and wire, and also on diffusion which for once may be benefical. Probably less than a fartor of 10 increase in the wire gain can be made before saturation becomes troublesome.

It is hard to judge what degree of puise symmetry can be realized in practice for the rather awkward sector shape, but the truth almost certainly lies between 1 part in $10^{2}$ and 1 part in $10^{3}$. Thus, the wire gain factor and the symmetry factor together provide perhaps only a factor of $10^{3}$, making the consideration of some additional steps necessary. The additional margin of safety could come from a gated feedback element in the wire preamplifier arranged to provide a low impedance path du: ing grid transitions. This concept is illustrated schematically in Fig. 7 which shows an FET switch enabling the preamplifier output section to reset large Induced charges appearing at the input. Clearly, the actual implementation of such a switch is a nontrivial design problem because the FET gate feedthrough can also induce large input charges. Here again, higher wire gain, by lowering the needed electrosic gain, reduces the design challenge significantly. 
The coupling of the grid to the sense wires is much greater than the grid-pad coupling due both to the small pad size and to screening effects of the sense plane, so that the pad preamplifiers probably do not require the gated feedback circuitry. Due to the broad $\mathrm{dE} / \mathrm{dx}$ spectrum, nois: requirements for wire channels are quite relaxed, relative to pad channels.

The margin of safety could be improved further by using closer wj re spacing in the grid. For example, by reducing the spacing hy a factor of two-thirds the two-lobed pattern in Fig. 6b would becom a three-lobed configuration. This helps in two ways; first, by reducing at least by a third the magnitude of the voltage needed to close the gate, and second, by reducing the effective "dipole soment" of the grid plane. Fven smaller grid wire spacing could be considered (i.e.. a four-lobed pattern inscead of three or two) but at some point the grid will begin to collect the primary elestrons even in the oren state. The threshold for electron collection (ignoring diffusion) may be calculated to a good approximation by conformal representation theory (9). For PEP-4 or !EF TPC sperating conditions, a four-lobed configuration should work, but this has not been experimentally verified. A four-lobed configurat on brings the grid closure voltages to the region of \pm 100 volts, a value accessible to current VMOS technnology. Complementary vNoS power FETs that switch an ampere in a few nanoseconds are available inexpensively and should serve well as the final elements in a grid pulser.

The voltages needed for grid closure would be I -duced somewhat further by reducing the drift region electric field. Other than the desire tc sheep out an entire drift volume prior to the arrivai of the next beam bunch, there is mo particular incentive for operating with high drift velocliy. The current LEP des., $n$ paraneters give 
25.5 useronds for $T_{b}$, the time iliterval between bunches, a very natural value for TPC techniques. Operation of LEP with four interaction regions rather than eight would increase this interval to 51 pseconds.

\subsection{Synchronous Gated Mode}

In this mode of operation, the gate cycles synchronously with bunch arrival, opening just before the bunches arrive and closing rapidly if a trigger is absent. The gate transition to the open state would be timed to allow for complete settling of iransients prior to bunch arrival. The synchronous mode thus allows the use of the complete drift region, whereas the asynchronous mode inevitably loses several cm to the processes of trigger development, gate transition, and settling times, of course, the presence of a valid trigger in the synchronous mode would cause the gate to stay open for the complete drift time. The positive ions generated in the readout planes are reduced, relative to an ungated mode, by the chopping action of the synchronous gate in a quadratic fashion. First, positive ions are generated only during the period $T_{c}$, defined as the interval between the bunch arrival and the gate closure; compared to the maximum drift time $T_{d}$ and assuming uniform ionization within the drift volume, only $T_{c} / T_{d}$ as many ions are generated as in the ungated case. $T_{c}$ may be as small as one microsecond. The ions generated arrive at the grid plane many $T_{b}$ intervals later, and although diffusion is small, the variations in path length create a smearing whith is long compared with $T_{b}$. Letting $T_{g}$ represent the entire effective "open gate" time (in the absence of event triggers), then approximately only $T_{g} / T_{b}$ ions are transmitted. A crude guess for $T_{g}$ is two microseconds. The return flux of positive ions is reduced by $F$, the product of 


$$
F=\frac{T_{c}}{T_{d}} \times \frac{T_{g}}{T_{b}}=\frac{1 .}{20.5} \times \frac{2 .}{25.5}=3.8 \times 10^{-3}
$$

This result for $F$ may even be conservative, as the effective open time $t_{g}$ for positive ions could be much smaller than the corresponding electronic interval. This situation would occur if the gate is closed "harder" than necessary, i.e., by using larger $\Delta v$ than minimum. A larger than minimum $\Delta v$ tends to create a zone of finite width that Ions must traverse to escape. As Ion drift velocities are on the order of $10 \mu \mathrm{m} / \mu \mathrm{sec}$, the zone need only be $100 \mathrm{\mu m}$ or less to effect nearly complete ion rejection for gate times of a few $\mu \mathrm{sec}$.

\subsection{Comparison of Asynchronous and Synchronous Modes}

A possible advantage of the synchronous technique is that the steady state of the system may be easier to tune to a high degree of symmetry and stability than the asynchronous mode. The pulsers in the synchronous mode will operate at $40 \mathrm{kHz}$ or $20 \mathrm{kHz}$, two orders of magnitude larger than the average asynchronous mode rate, but the power dissipation will still be only a few watts, at most per switching element. Much of the discussion regarding the grid plane configuration presented in the section on asynchronous operation is of course applicable to the synchronous mode, and the preceding discussion about hard gate closing is also of common relevance. It is also true that some of the discussion about gated mode performance is rather speculative and needs experimental work to verify expectations. Until further work is complete, even the choice between asynchronous or synchronous modes cannot be made with certainty. 
3. Miscellaneous Speculative Topics

\subsection{Gas Mixtures}

Argon-methane is perhaps unique as a TPC working gas. It displays extremely high electron mobility, unmatched by any mixture known to this author, and is very inexpensive and easy to purify to the requisite level. Nonetheless, the use of a ternary mixture of xenon-argonmethane may prove to offer a unique opportunity for 1mproving the particle identification efficiency. Xenon is regarded as having a higher relativistic rise than other gases, but the true behavior is still poorly known due to the presence in previous work of a substantial level of hydrocarbons such as propane, which can seriously limit the relativistic rise (4). It would be worthwhile to explore systematically the use of Argon-xenon-methane as a proportional ccunting gas, with methane limited to very low levels such as 2 or $3 \%$. The drift velocity would be reduced to 2 or $3 \mathrm{~cm} / \mu \mathrm{sec}$ but the additional pattern recognition problem of integrating backgrounds from more than one beam crossing $i$ not serious. In this connection it is interesting to note that studies by a Moscow group of this mixture at ultra-high pressure (100 atm) has shown that the addition of xenon can reduce by nearly a factor of two the anode voltage needed to reach a given gain, presumably by a high-pressure Penning effect. Gains in excess of $10^{4}$ were reached at these pressures even with $20 \mu \mathrm{M}$ diameter anodes $(10)$.

\subsection{Invariant Mass and Vertex Resolutions}

PEP-4 uses mylar rolled up to $-1 \mathrm{~cm}$ thickness as the insulating medium for the HV electrostatic system, and the resultant multiple scattering in this component, the inner drift chamber and the inner 
pressure vessel dominate the mass and vertex resolutions. It is conceivable to consider using a gas like $\mathrm{CO}_{2}-\mathrm{N}_{2}$ (even $\mathrm{SF}_{6}$ ) as the insulator, maintained at the TPC operating pressure but contained in a thin-walled gas-tight structure. Only a few cm are needed to contain $100 \mathrm{kV}$ at 7-10 atm with a properly shaped electrostatic system. The inner drift chamber of PEP-4 might not be necessary in the LEP environment, thereby liberating several radial $\mathrm{cm}$ for this purpose. It may also be conceivable to consider the use of beryllium instead of aluminum as the structural element for the inner pressure wall where particles enter the TPC active volume.

\subsection{Parallel Plane Geometry Replacing Sense Wires}

A conteivable readout plane geometry would be, in its simplest form, a grid or screen supporting an electric field intense enough to generate avalanches between itself and the pad plane (8). The electron avalanches would fall directly on the pad plane (now an anode plane), and the pad signals would necessarily carry both $d E / d x$ and spatial information. The track pair resolution in the $x$ direction may be improved considerably by this technique as the electron avalanche transverse size is of the scale of diffusion, i.e., one to two mu rms. Whether sufficiently stable gain for $d E / d x$ purposes could be achieved reliably with gases suitable for TPC drift lengths is an open question. The electronic shutter technique could also be realized by the use of an additional grid or screen plane just in front of the first screen, as in the multi-step chamber concept (8). 


\subsection{Calibration with Laser Beams}

Recently, groups at CERN have demonstrated the production of substantial iolization in typical MWP gas mixtures by intense iaser beams. Sufficiently narrow and well-surveyed beams should serve well as calibration standards in the drift volume of a TPC geometry as the sensitive volume presents no wires or surfaces to block the beams.

\section{Summary}

It appears that several modest improvements are straightforward possibilities in the application of the TPC concept at LEP. The successful development of a gated mode of operation would essentially eliminate the feedback of positive lons into the drift region. The TPC concept under these circumstances is a very natural technique for use in the LEP enviromment.

\section{Acknowledgment}

Many colleagues in the PEP 4 project have contributed to the ideas presented here, and $I$ wish to thank them for many interesting and valuable discussions. 
Table 1

GENERAL PARAMETERS OF THE PEP-4 TPC AND A HYPOTHETICAL TPC FOR LEP

\begin{tabular}{|c|c|c|}
\hline & PEP -4 & LEP \\
\hline Magnetic field $(T)$ & 1.5 & $1.5-2.0$ \\
\hline Inner sensitive radius (mn) & 200 & 200 \\
\hline Outer sensitive radius $(\mathrm{mm})$ & 950 & 1380 \\
\hline Number of sectors/end & 6 & 8 \\
\hline Longest sense wire $(\mathrm{mm})$ & 940 & 1060 \\
\hline Sense wire spacin's (mm) & 4.00 & 4.60 \\
\hline Sense wires/sector & 186 & 256 \\
\hline Total sense wires & 2232 & 4096 \\
\hline Grid wire spacing (mm) & 1.0 & $0.766-0.575$ \\
\hline Pad rows/sector & 15 & $12-16$ \\
\hline Pad size $\left(x y m^{2}\right)$ & $8.0 \times 8.0$ & $-5.0 \times 9.0$ \\
\hline Pads/sector & 1152 & $-1200-1800$ \\
\hline Total pads & 13824 & $-20,000-30,000$ \\
\hline Total drift length (mil) & 1000 & 1200 \\
\hline Maximum drift time (usec) & 17.9 & 20.5 \\
\hline Interval between bunches $T_{b}$ ( $\mu \mathrm{sec}$ ) & 2.41 & 25.5 \\
\hline Electron drift velocity (mm/usec) & 55.8 & 58.6 \\
\hline Operating pressure (atmospheres) & 10.0 & 7.6 \\
\hline Operating voltage (kv) & -100 & -100 \\
\hline Gas Mixture $\mathrm{ArZ}^{\mathrm{Z}}-\mathrm{CH}_{4} \mathrm{Z}$ & $85-15$ & $90-10$ \\
\hline
\end{tabular}


Table 1 (Continued)

$$
\text { PEP-4 }
$$

$\begin{array}{lcc}\mathrm{dE} / \mathrm{dx} \text { resolution (FWHM \%) } & 5.7 & 5.0 \\ \text { Amplifier shaping time (Gaussian FWHM ns) } & 200 & 200 \\ \text { Sample period in } \mathrm{Z}(\mathrm{ns} / \mathrm{MHz}) & 70 / 14 & 40 / 25 \\ \text { Sample length in } \mathrm{Z}(\mathrm{mm}) & 3.91 & 2.34 \\ \text { Spatial resolution In Z ( } \mathrm{m}) & -200 & 200 \\ \text { Spatial resolution in } \mathrm{x}(\mu \mathrm{m}) & \$ 200 & 200 \\ \text { Track pair interference zone }\left(\Delta x \Delta z \mathrm{~mm}^{2}\right) & 24 \times 21 & 15 \times 18\end{array}$


References

(1) The PEP-4 profect is a collaboration of groups from the Johns Hopkins University, Lawrence Berkeley Laboratory, University of Tokyo, University of Californta-Los Angeles, University of California-Riverside, and Yale University.

(2) Cork, B. and Hoang, T. F., LBL-9958 (1979).

(3) Allison, W. W. M. and Cobb, J. H., Oxford Untversity preprint $13-80$

(4) Walenta, A. H., Fischer, J., Okuno, H., and Wang, C. L., Nucl. Inst. and Meth. 161, 45 (1979).

(5) Fancher, D. L, and Schaffer, A.C., LBL-8327 (1978).

(6) Fancher, D., Hilke, H. J., Loken, S., Martin, P., Marx, J. N., Nygren, D. R., Robrish, P., Shapiro, G., Urban, M., and Wenzel, W., Nucl. Inst. and Meth. 161, 383 (1979).

(7) RCA, for example, is planning to make available in production volumes within one year a 6-bit $20 \mathrm{MH}_{2}$ flash encoder. Large volume prices are expected to be competitive with $\mathrm{CCD}$ costs. The developmental device number is TCS127.

(8) Breskin, A., Charpak, G., Majewski, S., Melchart, G., Peterson, G., and Sauli, F., CERN EP/79-01 and Nucl. Inst. and Meth. 161, 19 (1979).

(9) Buneman, 0., Cranshaw, T. E., and Harvey, J. A., Can, jour. of Res. 27A, 1:T (1949).

(10) Vishnevskii, A. V., Golutvin, I. A., Dolgoshein, B. A., Kalirovskii, A. N., Kaftanov, V. S., Sarantsev, V. L., Sviridov, v. A., Sosnovtsev, v. V., Khovanskii, v. D., Chernyatin, v. K., and Shevchenko, V. G., ITEP preprint 79-53. 
Figure Captions

Figure 1. Pad response as a function of avalanche position along a sense wire for PEP-4 geometry. Pad spacing is $8.0 \mathrm{mM}$ with a $0.5 \mathrm{mM}$ gap between pads, and $4 \mathrm{mM}$ between pad plane and sense plane. Response Is approximately Gaussian. Figure 2a. Position resolution in $x$ calculated from Gaussian form and $0.64 \%$ rns electronic noise relative to maximum pad signal.

Figure 2b. Scalloping of resolution is caused by large relative noise contribution when the avalanche is centered on a given pad, and neighboring pads have small signals. The resolution is Improved by using smaller pads as shown in the righthand figure.

Figure 3. Pad signal histogram for two tracks appearing simultaneously on a sense wire and separaced by three pad widths in $x$. The overlap is essentially negligible and the calculation of coordinate for each track can use three pads. For smaller separations where mixing of the two middle pad signals occurs, the tracks may be reconstructed well by using only the outer pad pairs.

Figure 4. Depiction of overlap criterion used for track pair resolution in 2 . Only a very small overlap occurs for a separation of eight sampling periods ( $320 \mathrm{nsec})$. Fitting techniques could easily resolve track pairs with considerably greater overlap.

Figure 5. Calculated momentum resolution for the parameter given in Table 1 for a LEP TPC. 
Figure 6a. Cross section of TPC readout plane showing field lines and - ectron trajectories (dashed lines) for an open gate.

Figure 6b. Samk geometry as Fig. 6a, but with alternate polarity voltages applied to the wires of the grid plane. The gate is closed by $\pm \Delta V$ of -200 volts. Electrons are collected on grid wires with $+\Lambda V$ and positive ions are collected on the grid wires with $-\Delta V$.

Figure 7. Symbolic preamplifiers with gated feedback element (an FET switch) to allow rapid resetting of transients. Other schemes to provide rapid overload recovery are certainly possible. 


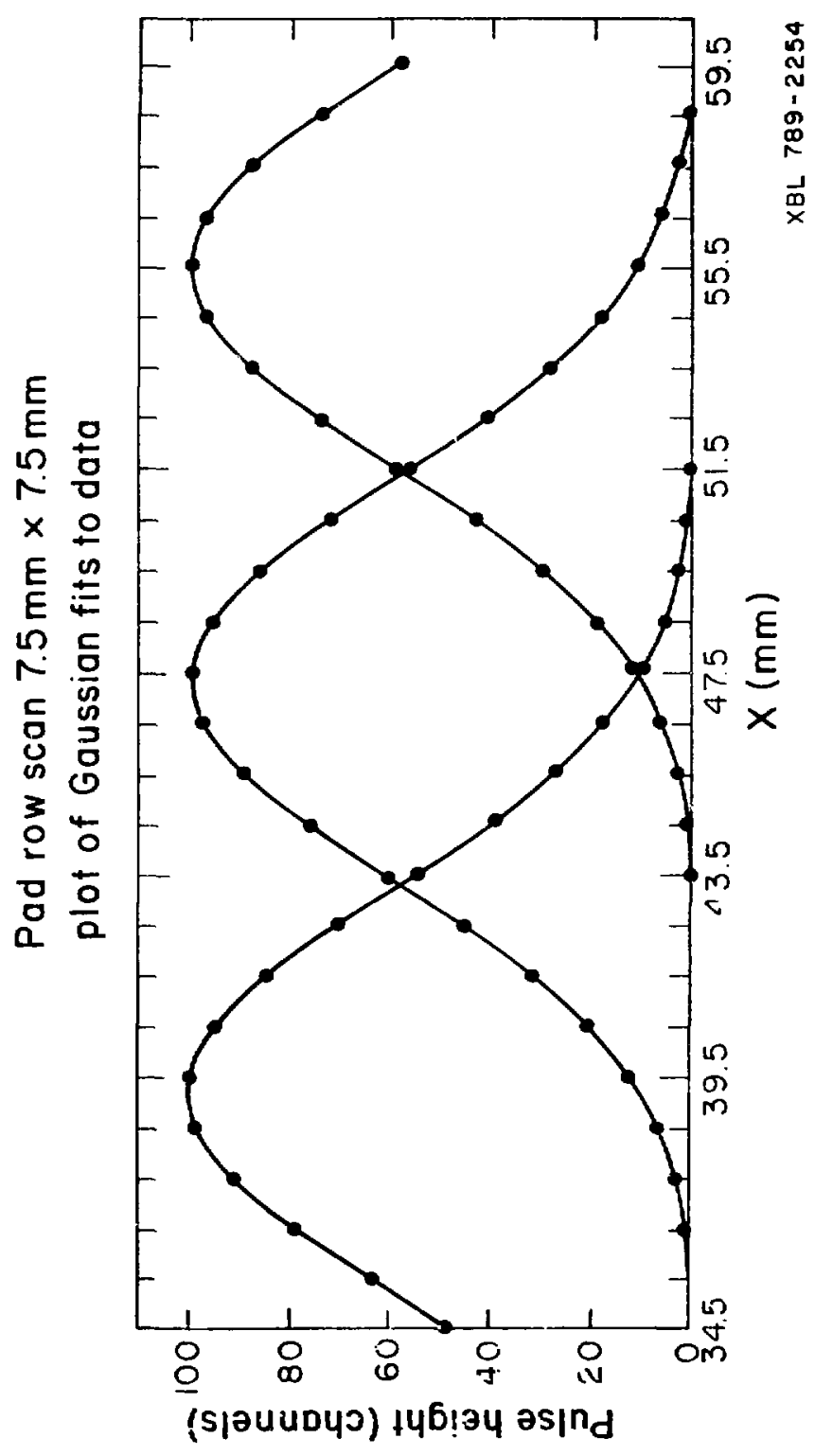



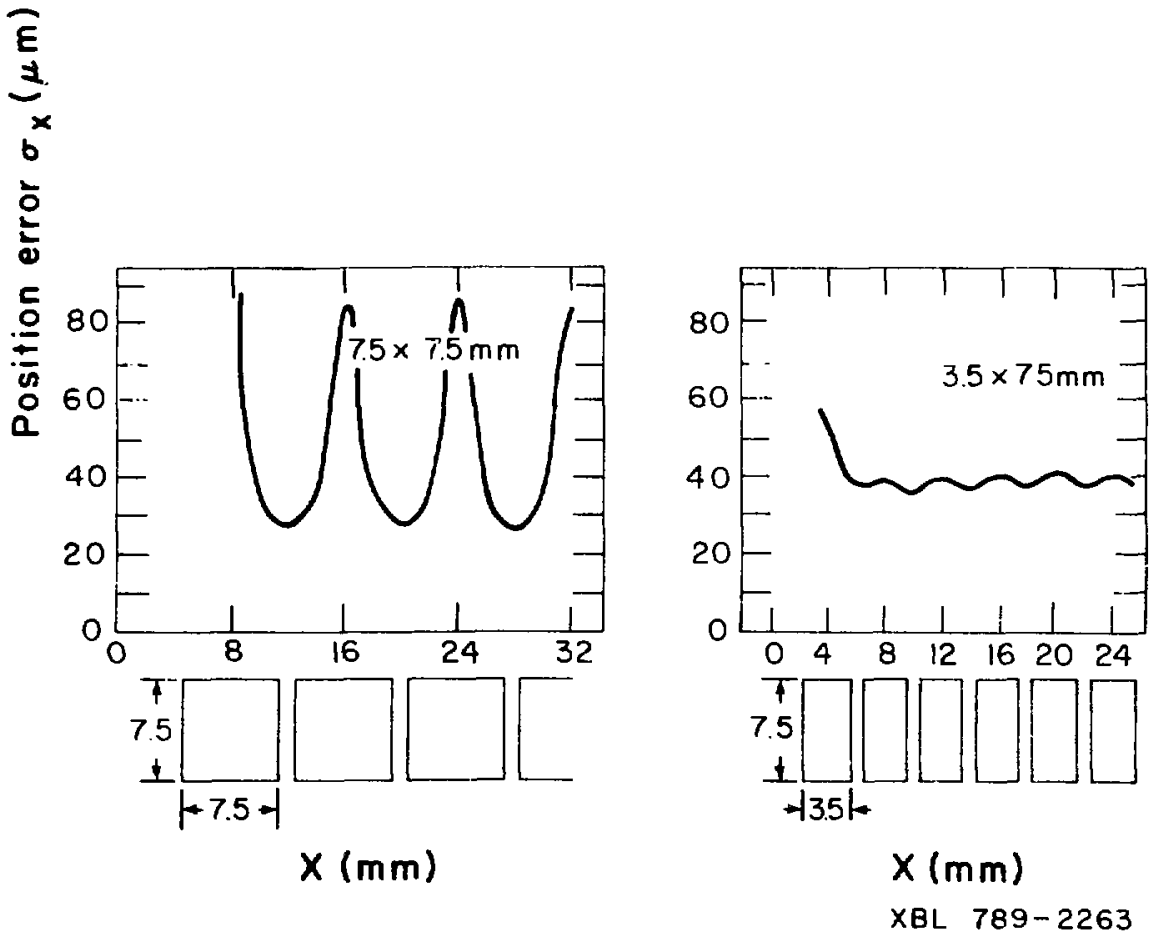

lig. ? 


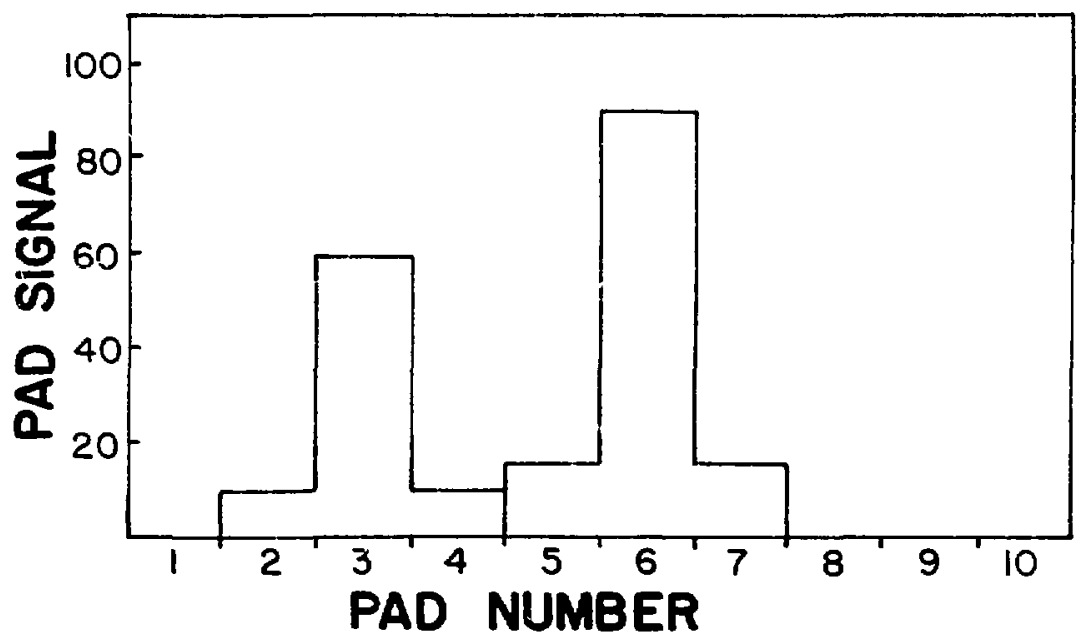

xBl. $80 \%-10655$

Fig, 3 


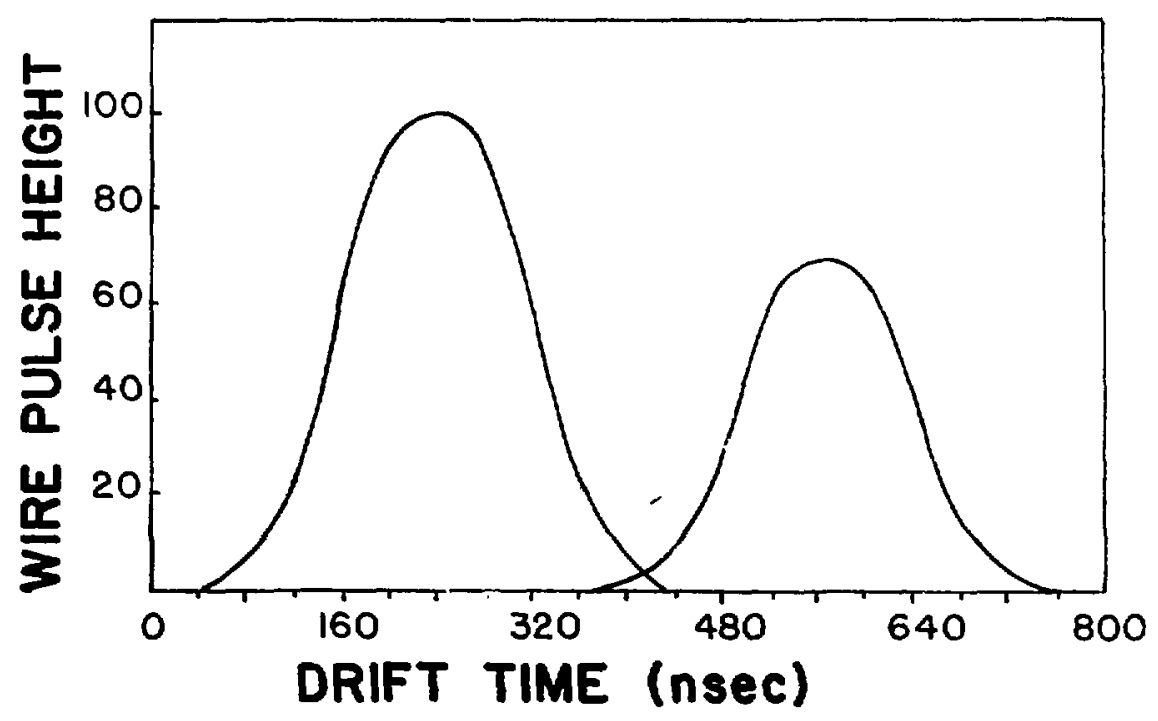

XBL. $807-10655$

Fir. 4 


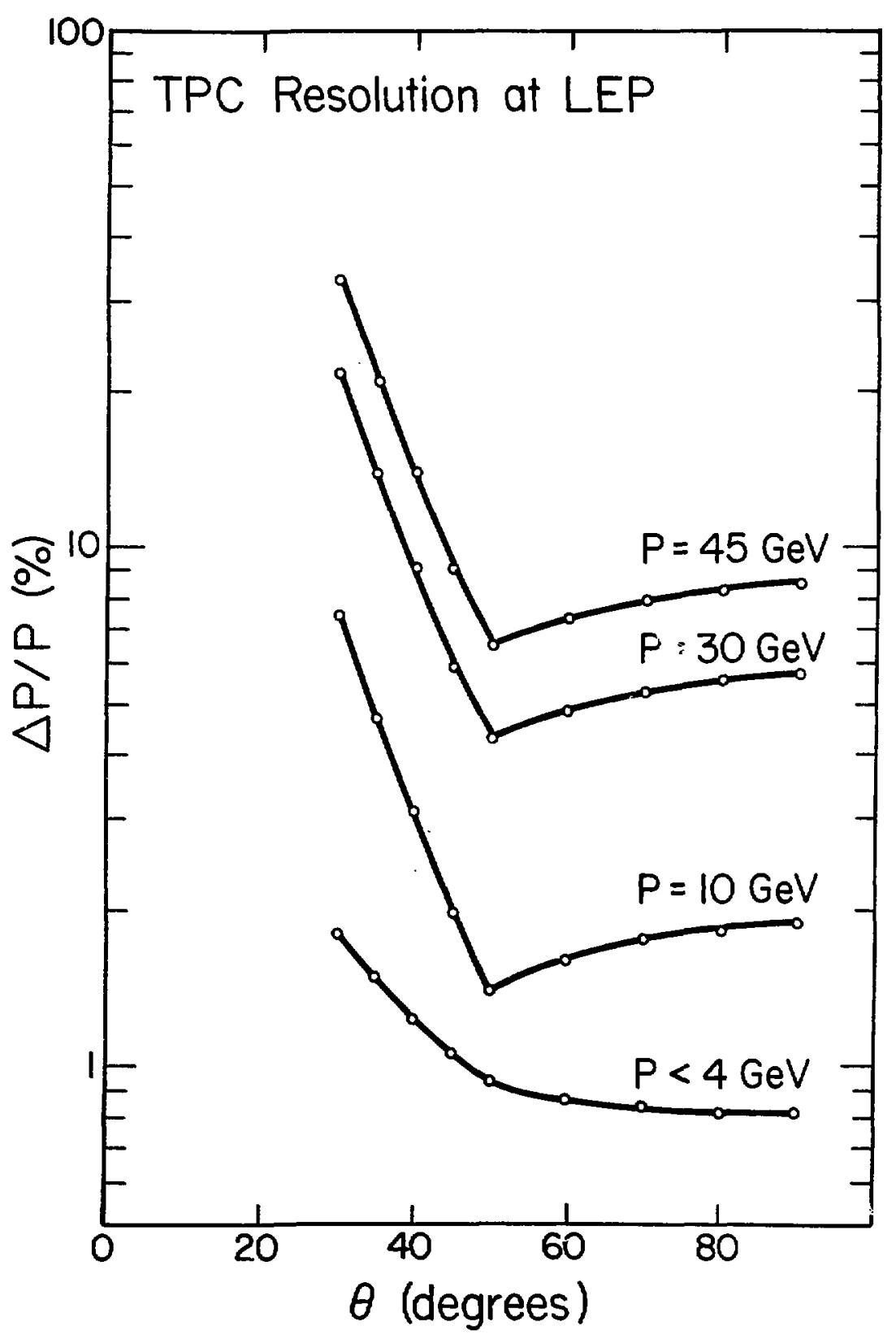

Fig. 5

XBL 806-1156 


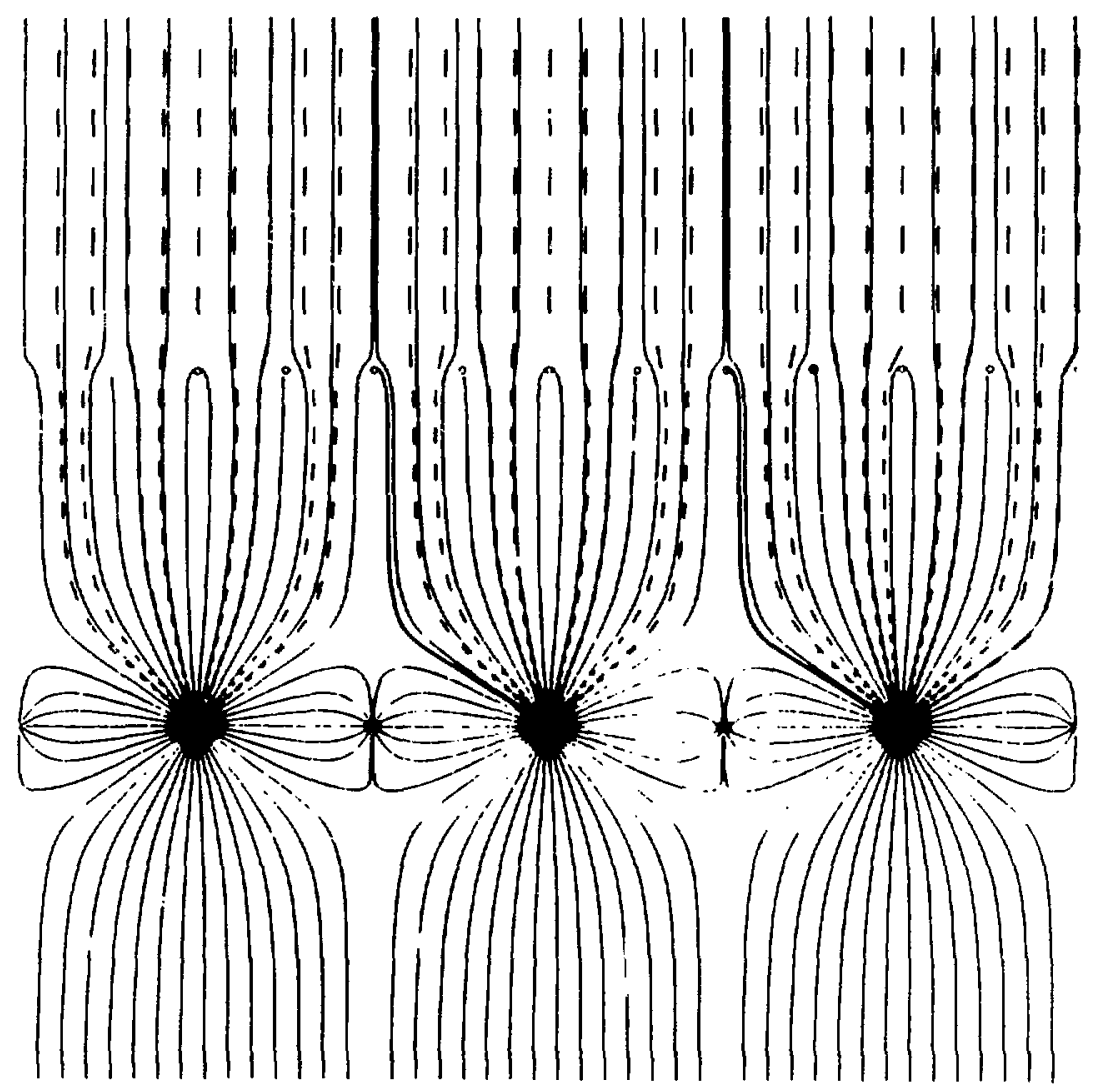

Ii ig. 6 a 


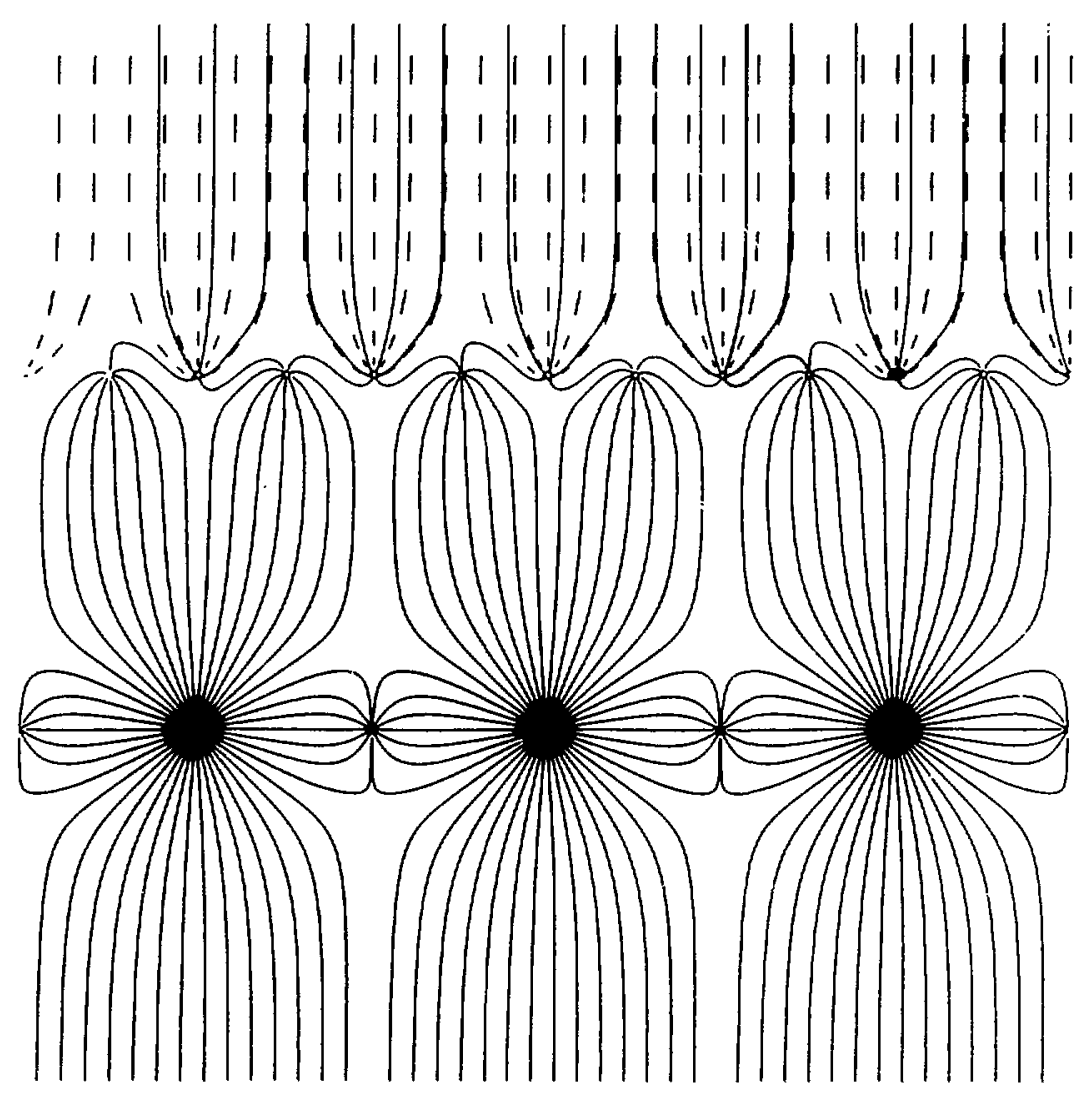

Iig. Gii

XBL $7810-11986$ 


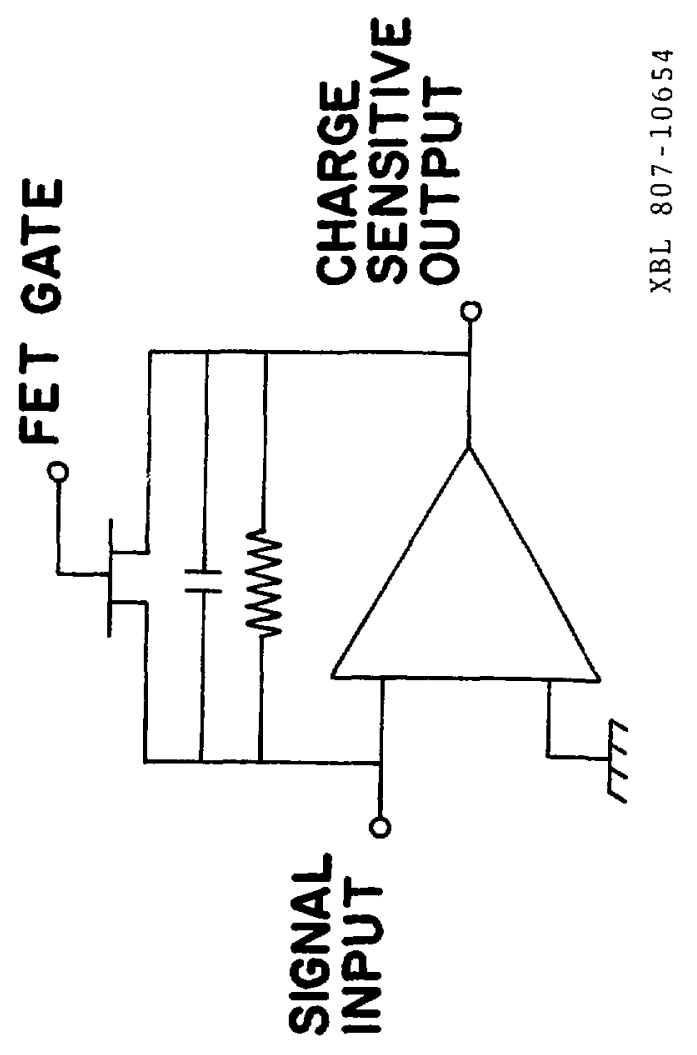

